\title{
COMPLICATIONS AFTER ARTHROPLASTY FOR BONE TUMORS
}

\author{
V.V. Protsenko ${ }^{1}$, V.F. Konovalenko ${ }^{2}$, V.S. Chorneyi ${ }^{3}$ \\ ${ }^{1}$ Institute of Traumatology and Orthopedics of NAMS of Ukraine, \\ ${ }^{2}$ Kavetsky Institute of Experimental Pathology, Oncology and \\ Radiobiology of National Academy of Sciences of Ukraine, \\ ${ }^{3}$ Bogomolets National Medical University, Ministry of Healthcare of \\ Ukraine Kyiv, Ukraine
}

\section{NTRODUCT ION}

The incidence of bone tumors is $0.5-2 \%$ per 100 thousand population. Arthroplasty is the main type of organ-sparing surgery in patients with bone tumors in $90 \%$ of cases. But at the same time, the effectiveness of arthroplasty is caused by complications that lead to repeated operations, including re-prosthetics. Revision endoprosthetics in primary oncologic endoprosthetics is about 30\% [1], and the risk of re-grafting in five years is $12-32 \%$, in 10 years - 25-61\% [2]. The literature reports that the main complications after arthroplasty are: infectious complications, aseptic instability of the endoprosthesis, a fracture of the endoprosthesis design, wear of the plastic parts of the endoprosthesis $[3,4,5,6]$. Thus, complications after joint arthroplasty is a significant problem that leads to dysfunction and sometimes loss of limb, significantly worsen the quality of life of patients who underwent joint arthroplasty for long tubular bone tumors.

\section{MATERIALS AND METHODS}

In the clinical departments of the State Institution "Institute of Traumatology and Orthopedics of the National Academy of Medical Sciences of Ukraine" for the period from 2009 to 2019,167 operations were performed for bone tumors in the volume of segmental bone resection followed by arthroplasty or re-prosthetics of the joints and bone segments. 110 patients received implantation of domestic oncologic endoprostheses, 57 patients received implantation of foreign modular endoprostheses. Morphologically, the following types of tumors were observed: giant cell tumor of the bone, osteogenic sarcoma, chondrosarcoma, fibrosarcoma and metastatic tumors. Replacement of the knee joint was performed in 95 patients, shoulder -22 , hip -20 , ulnar -9 , ankle -6 , diaphysis of the femur -5 , diaphysis of the tibia -4 , diaphysis of the humerus - 4, diaphysis of the radial bone -1 , hip and knee (megaprosthesis) - 1. During endoprosthesis replacement, the basic principles of oncological surgery radicalism and ablastic surgery were
Article history:

Received 26 February 2019

Received in revised form 17 March 2019

Accepted 02 April 2019

observed. Removal of the tumor was performed in one block with excision of the biopsy site with covering the endoprosthesis with surrounding muscles. For fixation of muscles, polymer tubes Mutars (Germany) were used, which increased tropism to soft tissues, which contributed to the rapid restoration of the natural muscle attachment point. Cement implant fixation was used for endoprosthetics. Pathological fractures were observed in 43 patients before surgery, 25 patients underwent various orthopedic surgeries before endoprosthetics, 74 patients underwent chemotherapy before surgery, 30 patients underwent radiation therapy before surgery, which played an important role in the occurrence of postoperative complications. In all cases, endoprosthetics were used to measure the volume of the resected bone segment. The length of the resected bone segment, the duration of anesthesia and blood loss during the operation were taken into account, which also influenced the development of postoperative complications. The functional result of the operated limb was calculated on the MSTS scale. The quality of life of patients was determined by points according to the EORTC QLQ-30 questionnaire. Patient survival was determined by the Kaplan-Meier method.

\section{RESULTS}

After primary oncologic endoprosthetics, 51 $(30,5 \%)$ patients experienced complications in different postoperative periods, $37(22,2 \%)$ of them underwent re-endoprosthetics. The most frequent complications after arthroplasty were the following: infectious -24 cases, aseptic loosening of the endoprosthesis leg -21 , fracture of the endoprosthesis design -5 , wear of the polyethylene parts of the endoprosthesis -1 . Recurrences of the tumor were observed in 13 (7,8\%) patients. The functional outcome of the limb (on the MSTS scale) after hip joint arthroplasty was $80 \%$, femur diaphysis $94 \%$, knee joint $84 \%$ of the tibial diaphysis $92 \%$, ankle joint $70 \%$, shoulder joint $70 \%$, diaphysis of the humerus - $96 \%$, of the elbow joint $78 \%$, diaphysis of the radial bone - 90\%, hip and knee (megaprosthesis) - 72\%. The quality of life of patients after arthroplasty has increased from $40-50$ to $75-80$ points. The total three-year survival of patients was $82,2 \pm 0,14 \%$, the five-year one $-65,8 \pm 0,26 \%$. 


\section{CONCLUSIONS}

The main complication factors after arthroplasty in patients with bone tumors were the following: previous surgery, previous chemotherapy or radiation therapy, tumor localization, tumor volume removed, length of resected bone fragment relative to bone length, duration of surgery, and amount of blood loss during surgery, implant design and implant installation technique.

\section{REFERENCES}

1. Aseptic loosening is uncommon with uncemented proximal tibia tumor prostheses / M.N. Flint, A.M. Griffin, R.S. Bell [et al.] // Clin. Orthop. Relat. Res. 2006. - Vol.450. - P.52-59.

2. Endoprosthetic replacement of the distal femur for bone tumors: long-term results / G.J. Myers, A.T. Abudu, S.R. Carter [et al.] // J. Bone Joint Surg. Br. 2007. - Vol.89 (4). - P. 521-526.
3. SINGH J.A. Epidemiology of knee and hip arthroplasty: a systematic review / J.A. Singh / Open Orthopaedics J. - 2011. - Vol. 5, No 1. - P. 80-85.

4. SKuteK M. International epidemiology of revision THR / M. Skutek, R.B. Bourne, S.J. Mac Donald // Orthoped. Trauma. - 2006. - Vol. 20, No 3. - P. 157-161.

5. The epidemiology of revision total hip arthroplasty in the United States / K.J. Bozic, S.M. Kurtz, E. Lau [et al.] // J. Bone Jount Surg. Am. - 2009. - Vol. 91, No 1. - P. 128-133.

6. Use of (18) F-FDG-Pet in the diagnosis of endoprosthetic loosing of knee and hip implantants / M. Schunert, S. Mayer-Wagner, W. Mayer [et al.] // Arch. Orthop. Trauma Surg. - 2010. - Vol. 130, No 10. - P. 1231-1238 